and the manufacture of drugs. Various approaches are being adopted to conserve horseshoe crabs. The Ocean Park Conservation Foundation and City University of Hong Kong have been running a juvenile horseshoe crab rearing programme in Hong Kong since 2010, to promote the importance of horseshoe crab conservation among secondary school students and the public. Scientists and conservationists in Taiwan have been promoting an integrated conservation strategy, encompassing research, artificial breeding and field release, public education and establishment of a protected area for horseshoe crabs. In mainland China, artificial breeding and field release of juvenile horseshoe crabs have been carried out to enhance wild populations. In 2013 the FFI China Programme initiated a project to promote participatory monitoring of juvenile horseshoe crabs in Beibu Gulf and strengthen the conservation network within China, of which the workshop was part.

With support from all attendees, from 22 institutions and organizations, a regional Horseshoe Crab Conservation Consortium was born. The Consortium has a mission to (1) promote the conservation status of horseshoe crabs, (2) reduce illegal utilization and trade of horseshoe crabs and promote their sustainable use, (3) promote the establishment of protected areas for horseshoe crabs and enhance effective management and habitat restoration, (4) develop research and conservation capacity and strengthen sharing of information, and (5) raise the awareness of the government, public and other stakeholders regarding the conservation of horseshoe crabs. The Consortium set up four working groups to promote efforts for population monitoring and Red List assessment, artificial breeding and field release to enhance wild populations, public education, and wise use of horseshoe crabs for societal needs. The aims of the Consortium are intended to extend beyond China and to facilitate collaboration among scientists and conservation practitioners from other Asian countries that host horseshoe crab populations.

WUYING LIN Fauna \& Flora International China Programme, Beijing, China. E-mail linwuying@ffichina.org

Chang-Po Chen Taiwan Wetland Society and Academia Sinica

PAUL SHIN IUCN SSC Horseshoe Crab Specialist Group and City University of Hong Kong

\section{Bogus captive breeding of oriental rat snakes}

In March 2014 the Indonesian Ministry of Forestry announced they had given permission to two East Javan companies to export captive-bred rat snakes specifically as pets, with an annual quota of 1,850 Ptyas mucosa allocated to the Kediri-based UD Bina Usaha Mandiri and 125,000
Ptyas korros and 50,000 P. mucosa allocated to the Sidoarjo-based CV Karya Albadi Reptil Mulia. These quotas are considerably larger than previous quotas of up to 3,000 for P. mucosa and 1,00o for P. korros. Both companies are large-scale exporters of dried geckos and snakes to East Asia, and there are no indications that either of them have ever been involved in the trade of live animals or that they are experienced in breeding reptiles. Auliya (2010, Conservation Status and Impact of Trade in the Oriental Rat Snake in Java, TRAFFIC, Petaling Jaya) gives a detailed account of the trade in rat snakes in Central and East Java, confirming the absence of their trade for pets in or from Java, and including data on reproduction, growth rates, and prices. These data allow us to evaluate whether captive breeding of rat snakes is economical.

Rat snakes are predominantly terrestrial and on Java can be found in a range of habitats, and are abundant in agricultural areas such as rice fields. The snakes are collected in large numbers either opportunistically by farm workers or by professional harvesters, and passed on to middlemen who sell them to large-scale traders. International trade in P. mucosa is regulated through CITES, with $>90 \%$ of the trade comprising skins and meat. After having been banned from exporting P. mucosa for 12 years, Indonesia now sets an annual export quota of 90,000 wild-caught individuals for P. mucosa $(89,500$ to be exported for their skins, 500 as pets) and 4,500 wild-caught individuals for $P$. korros (1,800 skins, 2,700 pets) but captive-bred individuals can be traded above and beyond this quota.

A rat snake's value increases with size, with the maximum values attached to skins of $140 \mathrm{~cm}$ length and live snakes of $800 \mathrm{~g}$ or more; at these sizes rat snakes have retail values for Indonesian exporters of USD 2.85-2.96 when sold alive and USD 3.03 when the skin and meat are sold separately.

Oriental rat snake females become sexually mature in 9 months, gestation and incubation last 5 months, and mean clutch size on Java is 15 eggs. Males and females have slightly different growth rates but they reach their economically optimal size in 15-20 months. If under optimal conditions females can produce two clutches per year, all 30 eggs hatch and there is no mortality of the young, then to produce 176,850 rat snakes per year one has to maintain a breeding stock of almost 4,00o adult females.

Given the low price rat snakes command and their apparent abundance, especially in Central and East Java, it cannot be economical to breed rat snakes for commercial purposes. Maintaining breeding stock, taking care of eggs and feeding young for over a year, only to be able to sell them on for $<$ USD 4 , suggests that wild-caught individuals are probably being exported as captive-bred to circumvent the quota system for wild-caught individuals. I urge the Indonesian CITES Scientific Authority, which sets these quotas, to reconsider its decisions and for importers to 
carefully check whether or not animals (or their parts) declared as captive-bred have indeed been bred under controlled conditions.

VINCENT NIJMAN School of Social Sciences and Law, Department of Anthropology and Geography, Oxford Brookes University, Oxford, UK

E-mailvnijman@brookes.ac.uk

\section{Photographic database informs management of conflict tigers}

The increasing interface between people and wildlife contributes to human-wildlife conflict in many conservation landscapes. In India animals suspected to be involved in conflict are often captured and translocated to different locations or zoological parks. A key concern in the capture of so-called problem animals has been the identification of individuals involved in conflict. The Wildlife Conservation Society (WCS) India Program, in collaboration with research partners, has aided government officials in this matter through its research on tigers in the Malenad Tiger Landscape, south-west India. As part of a research programme initiated in 1991, WCS India has formulated a protocol for individual identification of tigers, based on their stripe patterns. Using pattern-matching software that aids quick and reliable identification of tigers from photographs (Hiby et al., 2009, Biology Letters, 5, 383-386), a photographic database has been developed, with $>750$ individually identified tigers from 16 protected areas totalling $>38,000 \mathrm{~km}^{2}$, and elsewhere, in India. Ancillary information on home ranges, activity patterns and sociobiology of conflict-tigers provides a unique opportunity for informed management of the species. This long-term, ongoing study is funded by the Department of Science and Technology and the Department of Biotechnology of the Government of India, Vision Group on Science \& Technology of the Government of Karnataka, and the Wildlife Conservation Society, New York.

Two recent cases of human-tiger conflict in the Malenad Tiger Landscape exemplify the utility of this database and long-term monitoring for conflict mitigation. One incident, on 27 December 2013, involved the loss of human life in Bandipur National Park, and the other, on 1-2 January 2014, involved the killing of cattle in a village adjoining Nagarahole National Park. In the latter incident a dead cow was used as bait to capture the tiger involved. In the incident in Bandipur photographs from camera traps set up in the area of conflict were matched to individuals in the database. One individual was identified in nine of the 15 photographs obtained and, as the location of conflict was well beyond its home range, it was identified as the conflict individual and was subsequently captured. The tigers from both incidents have been transferred to a nearby zoo. The Forest Department debated relocation of the individuals but this was not pursued.

Both of the tigers involved in these incidents had been photographed previously multiple times. The tiger in Bandipur had been photographed over the last 5 years and was probably an old individual past its prime. The tiger in Nagarahole was 2-3 years old and had only been photographed in the previous year. The distances between the locations of previous photographs and the location of conflict were $4-8 \mathrm{~km}$ for the tiger in Bandipur and $35 \mathrm{~km}$ for the tiger in Nagarahole. The observation of the Nagarahole individual with another male in two photographs suggests that this individual was dispersing from its natal home range to establish a breeding territory.

Nagarahole and Bandipur National Parks have high tiger densities (10-15 tigers per $100 \mathrm{~km}^{2}$ ) with c. $20 \%$ of the population lost annually to mortality and emigration. It is likely that the Nagarahole individual was emigrating from the reserve. The infrequency of dispersal events, particularly in human-dominated landscapes such as the Malenad Tiger Landscape, suggests that the removal of the Nagarahole tiger, a potential long-distance disperser, may have detrimental effects on the long-term population viability of tigers in this landscape. In contrast, the removal of the Bandipur tiger will probably have negligible effects on the population, although any relocation into an existing tiger population would be likely to cause further conflict (Athreya et al., 2011, Conservation Biology, 25, 133-141). Mitigation of conflict is frequently ad hoc, with management authorities pressurized into quick but often ineffective action. In these two contrasting examples, alternative actions could have had very different consequences. In areas where data on the age, reproductive status and ranging patterns of conflict animals are available, this information should be used to direct conflict mitigation strategies such that they are maximally effective whilst inflicting least harm on species viability.

K. Ullas Karanth, N. Samba Kumar and Divya VasudeV Wildlife Conservation Society, India Program, Bangalore, India. E-mail vasudev.divya@gmail.com

\section{First village successfully resettled from Dandeli-Anshi Tiger Reserve}

Much debate on the conservation of species such as the tiger has centred around the need for inviolate spaces devoid of human presence. A conservation strategy that emerges from this is the resettlement of villages from within the core zones of tiger conservation areas. The resettlement of such villages not only frees up space for dedicated tiger conservation but provides the resettled people with better access to amenities and serves to mitigate human-wildlife conflict (Karanth, 2007, Biological Conservation, 139, 315-324). Although 